\title{
The role of complexions in metallic nano-grain stability and deformation
}

Timothy J. Rupert ${ }^{1,2, *}$

\begin{abstract}
Nanocrystalline metals have excellent strength due to the high density of grain boundaries inside. However, these same boundaries lead to limited thermal stability and a tendency to fail in a brittle manner, issues which limit the practical usage of these materials. Most strategies for stabilization of nano-grains against coarsening rely on the idea of using segregating dopants to lower excess boundary energy. The theory of interface complexions is a useful tool for describing the thermodynamics behind segregation as well as identifying distinct segregation patterns. Some of these same complexions can also dramatically alter mechanical behavior. Unlike past strategies, which always result in a trade-off between strength and ductility, the addition of complexions can potentially increase ductility while retaining or even increasing strength. In this paper, we discuss how complexions offer a unique opportunity to address these limitations simultaneously. In addition to reviewing the current-state-of-the-art, important areas where innovation is needed are also identified.
\end{abstract}

Keywords: Nanocrystalline materials; Thermal stability; Mechanical behavior; Complexions; Grain boundary; Segregation; Intergranular films; Atomistic modeling 


\section{Introduction and Motivation}

Nanocrystalline metals are defined as having an average grain size $(d)$ less than $100 \mathrm{~nm}$. This dramatic grain refinement brings an equally dramatic increase in the volume fraction of material that is located at the grain boundaries, with the geometric analysis of Palumbo et al. [1] showing that roughly $30 \%$ of the material is located in the grain boundaries for an average grain size of $10 \mathrm{~nm}$. With this rising grain boundary volume fraction comes a large increase in strength, often greater than an order of magnitude higher than the strength of microcrystalline metals. For example, coarse-grained $\mathrm{Cu}$ only exhibits a yield strength of $\sim 50 \mathrm{MPa}$ [2], while the yield strengths of nanocrystalline $\mathrm{Cu}$ samples with grain sizes of $d=26 \mathrm{~nm}$ and $d=30 \mathrm{~nm}$ were reported to be $535 \mathrm{MPa}$ [3] and $760 \mathrm{MPa}$ [4], respectively. This extreme strengthening has been observed for many metallic systems, often opening new avenues for the use of a particular material. For example, $\mathrm{Al}$ is an extremely light metal but tends to be weak in its pure form. Elemental $\mathrm{Al}$ is not widely used as a structural metal, with heavy alloying required to increase strength to an acceptable level even for use in aerospace applications, where limiting weight is the major priority. On the other hand, pure $\mathrm{Al}$ can be strengthened to levels comparable to steel alloys by grain refinement to the nanometer range [5]. Other advantages of nanostructuring include increased resistance to wear [6,7] and fatigue [8]. However, the two major limitations of nanocrystalline materials can also be connected to the preponderance of grain boundaries: (1) limited thermal stability and (2) lack of ductility.

Grain boundaries, like all defects, have an excess free energy associated with the deviation from the preferred crystalline arrangement of atoms. Nanocrystalline metals therefore have a very large driving force for coarsening of the grain structure, in order to reduce the interfacial area inside the material. As a result, pure or elemental nanocrystalline metals are 
typically unstable and rapidly coarsen $[9,10]$, with significant grain growth sometimes occurring even at room temperature [11]. Loss of the nanostructure means that the targeted high strength will be lost as well, making the stabilization of nano-grains at service temperatures a key goal in this field. In addition, this lack of thermal stability has an important implication for the practical production of nanocrystalline metals. Processing routes for bulk nanostructured materials can be loosely broken into "top down" techniques, where a coarse-grained microstructure is refined (usually by severe plastic deformation), and "bottom up" techniques, where small quantities of material are assembled to create a bulk material. Unfortunately, although it is relatively simple to create bulk materials with top down techniques, these methods are often limited to refinement into the so-called ultrafine grain regime, with grains less than $1000 \mathrm{~nm}$ but larger than $100 \mathrm{~nm}$. Alternatively, bottom up techniques, such as ball milling powder samples, can reduce grain size to $\sim 20 \mathrm{~nm}$ in many cases, but require a consolidation step for the fabrication of bulk pieces. Since consolidation requires the application of a high temperature, typically $>50 \%$ of the melting temperature, increased thermal stability is also required to enable the scalable production of nanocrystalline metals [12].

Nanocrystalline metals have extremely high strength, but this is almost always accompanied by a dramatic decrease in ductility. For example, nanocrystalline $\mathrm{Cu}$ created by surface mechanical attrition only exhibits a total plastic strain-to-failure of 3\% [4], an extremely low value for a metal. A general trend is observed where strength and ductility of nanocrystalline metals are mutually exclusive properties [13]. One can either have a strong yet seemingly brittle response, or a soft and ductile behavior. For example, Gianola et al. [14] demonstrated that mechanically-induced grain boundary migration can give nanocrystalline thin films additional ductility when this mechanism is activated, but these samples are much weaker 
than expected. Fracture surfaces from nanocrystalline materials show evidence of dimpled rupture [15], a signature that the nano-grains themselves are able to sustain plasticity. However, this intrinsic ductility of each nano-grain does not translate to macroscopic or extrinsic ductility. Appreciable ductility is required of most structural materials, so that catastrophic failure is avoided. Although yield is typically defined as a failure, it is preferred that a component deforms irreversibly yet survives and can still carry load, as opposed to shattering or crumbling soon after yield. A ductile response is also helpful for processing tasks, where shaping into a final form often requires plastic flow.

In this article, we discuss the current state-of-the-art strategies used to address the limited thermal stability and ductility of nanocrystalline metals, with an eye toward exploring how the theory of complexions can be useful for understanding important behavior and enabling further developments. We first provide a brief overview of some of the important aspects of complexion theory that are especially relevant to this discussion. We then focus on the two challenges of thermal stability and mechanical behavior one at a time, isolating the important physical phenomena behind each limitation and identifying opportunities for innovation in both areas. As these behaviors are intimately related to grain boundaries, direct control over interfacial structure, chemistry, and properties should have a dramatic impact. The unique opportunity offered by complexion engineering is not just that it can address these issues, but rather that one material design methodology may be able to simultaneously address both.

\section{Complexions: Distinct Interfacial States}

Materials scientists have long acknowledged that grain boundaries can be thought of as two dimensonal "phases-like" features which can undergo structural transitions at critical values 
of temperature or chemical composition [16-18]. The term complexion, denoting interfacial material that is in thermodynamic equilibrium and has a stable finite thickness, has recently been adopted in the literature [19-21]. Complexions do not technically adhere to the strict definition of a phase introduced by J.W. Gibbs since their structure depends on the orientation and chemistry of the neighboring crystallites [22]. The concept of distinct and thermodynamicallystable interfacial structures can be traced back as far as 1860 when surface premelting was proposed by Faraday [23]. Unfortunately, excitement for such an idea waned over time, as it became clear that complexions were exceedingly rare and difficult to access in single element systems. For example, Hsieh and Balluffi [24] performed in situ transmission electron microscopy (TEM) heating experiments and found that grain boundaries in pure Al do not premelt even at temperatures as high as $0.999 T_{\text {melting. }}$ However, recent efforts have shown that these interfacial complexions become much more common and accessible in multicomponent systems [25-28].

Dillon, Harmer, and coauthors recently created a categorization scheme that relies on complexion thickness based on high-angle annular dark field scanning TEM observations in doped $\mathrm{Al}_{2} \mathrm{O}_{3}$ [19]. Six discrete Dillon-Harmer complexions were found, as shown in Fig. 1, with each displaying a different grain boundary mobility as measured by grain growth kinetics. These include: (I) a single layer of dopants, (II) clean grain boundaries, (III) bi-layers, (IV) multilayers, (V) nanoscale films of equilibrium thickness, and (VI) wetting films. All of the structures shown, with the exception of the wetting film which is actually a bulk phase sandwiched between two new complexions (the interfaces between the original crystals and the new film), have a thickness which is determined by thermodynamics. While reports of complexions are 
much more common in materials with directional bonding such as ceramics [29], analogous examples of similar complexions have recently been reported in metallic systems [20,30,31].
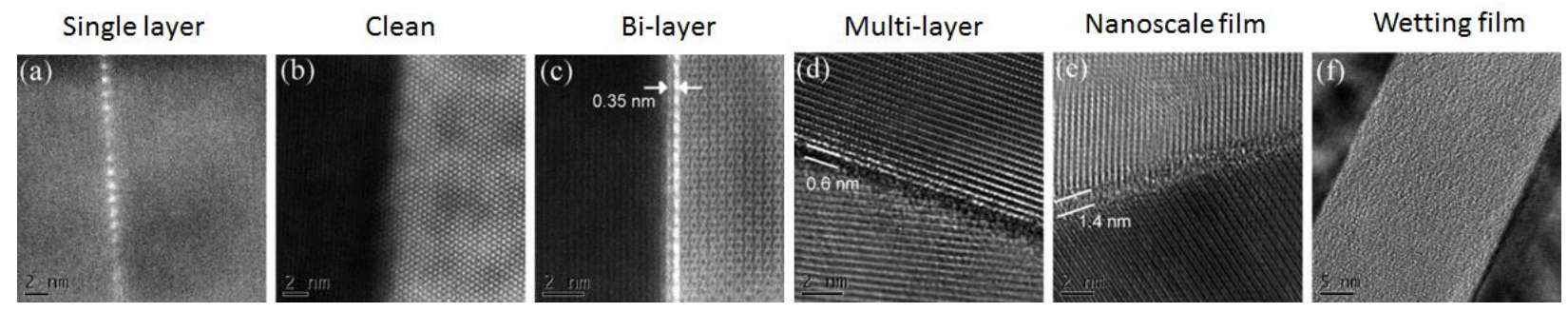

Fig. 1. High-angle annular dark field scanning TEM images show the variety of complexion types that can be observed in doped $\mathrm{Al}_{2} \mathrm{O}_{3}$. These include (a) single layer segregation, (b) clean grain boundaries, (c) bi-layer segregation, (d) multi-layer segregation, (e) nanoscale films with equilibrium thickness, and (f) wetting films. Different degrees of structural and chemical disorder can be found with this variety of complexions. Reprinted from [19], with permission from Elsevier.

The Dillon-Harmer complexion types are only one of many possible categorization schemes. A number of other options exist, with characteristics like complexion geometry, composition, and even changes to misorientation or boundary normal used a differentiating features. However, any scheme includes simplifications of some kind that cannot capture all possible degree of freedom, meaning multiple variations are often necessary for a complete description. For a discussion of nano-grain stability and mechanics, we will rely heavily on the Dillon-Harmer classifications but it is also important to consider the periodicity found within different complexions. Complexions can either have a recognizable degree of periodicity (ordered) or a lack of long-range order (disordered) in either structural units or chemical composition. For example, complexion Types I-IV above would be structurally ordered, as atoms are arranged in a set number of layers. On the other hand, Types V and VI can be either structurally ordered, with a crystalline film forming, or disordered, with an amorphous/glassy structure to the film. Chemical order is less closely tied to the six Dillon-Harmer complexion types, as all except Type II (which has only one element and is therefore pure) can have adsorbates that are arranged either periodically or non-periodically. In many cases, these 
distinctions have important implications for the properties of the interfaces and, therefore, the material as a whole.

\section{Thermal Stability of Nano-grains}

The lack of thermal stability in nanocrystalline materials can in theory be addressed by either thermodynamic or kinetic stabilization of the microstructure. The migration of a grain boundary can be understood by looking at the simple relation introduced in Eq. 1 [12]:

$$
v=M \times P=M_{0} \exp \left(\frac{-Q_{m}}{R T}\right) \times \frac{2 \gamma}{r}
$$

where $v$ is the curvature driven velocity of a grain boundary during grain growth, $M$ is the grain boundary mobility, $P$ is the driving force for grain growth, $M_{0}$ is a mobility constant, $Q_{m}$ is an activation energy, $R$ is the gas constant, $T$ is temperature, $\gamma$ is grain boundary free energy per unit area, and $r$ is the radius of curvature of the interface. Thermodynamic strategies for increased thermal stability focus on reducing the driving force for grain growth, or the grain boundary free energy. Nearly all thermodynamic nanostructure stabilization theories can trace their roots to the seminal work of Weissmuller [32], who suggested that the energy reduction associated with dopant segregation could reduce the grain boundary energy to zero and completely remove the driving force for grain growth. On the other hand, kinetic strategies try to dramatically reduce the mobility term, often by adding solutes or second phase particles which restrict the moving interface $[33,34]$. Of the two, the thermodynamic approach is often preferred because it can in principle remove the problem of grain growth altogether. Kinetic approaches always break down at some point, as high temperatures or long times at temperature will inevitably lead to the loss of nanoscale grain structure. 
In the original formulations by Weissmuller [32,35], a McLean model for segregation, which assumes dopants can only fill a single monolayer of sites, was used to describe the total Gibbs free energy of a nanocrystalline alloy. With a strongly segregating dopant species added to the nano-grains, it is possible to minimize the free energy of the alloy with respect to grain boundary area. In other words, a thermodynamically stable grain size that depends on alloy composition can be achieved. However, such early models came with relatively strict theoretical assumptions, such as requiring that grain boundaries be saturated by dopant atoms and that grain interiors be dilute. Trelewicz and Schuh [36] worked to relax such assumptions by developing a nanocrystalline regular solution approach that included both grain interior and grain boundary regions. Saber et al. [37] later developed a similar regular solution model and made predictions for nanocrystalline $\mathrm{Fe}-\mathrm{Zr}, \mathrm{Cu}-\mathrm{Zr}, \mathrm{Cu}-\mathrm{Nb}$ and $\mathrm{Ni}-\mathrm{W}$. A common feature of these models is that they actually describe metastable nanocrystalline structures, as they do not compare the nanocrystalline state with competing bulk phases. Murdoch and Schuh [38] added the possibility of phase separation, limiting their attention to alloys with positive enthalpies of mixing to simplify the problem, while Zhou and Luo [39] considered both second phases and the possibility of multi-layer dopant segregation at the boundaries. Fig. 2(a) shows how such models can be used to create a Gibbs free energy surface to identify the boundary concentration and grain size associated with a stable nanocrystalline grain structure [38]. All of the methods described above are extremely useful for identifying candidate binary element combination that can be made into thermodynamically-stable nanocrystalline metals. For example, Darling et al. [40] performed a large-scale computational study where over 1000 binary systems were analyzed to identify promising alloys. Fig. 2(b) shows an example from this work for nanocrystalline $\mathrm{Fe}$ with a variety of potential dopants. 
(a)

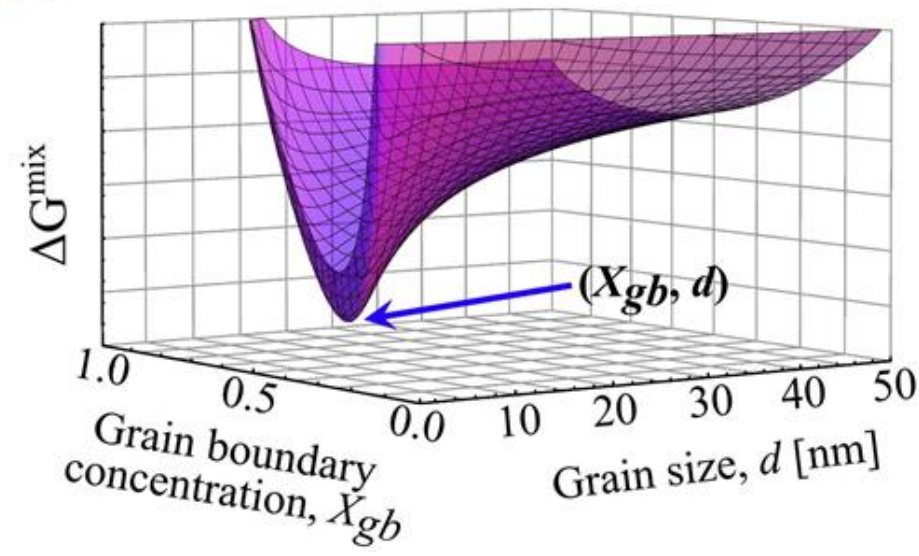

(b)

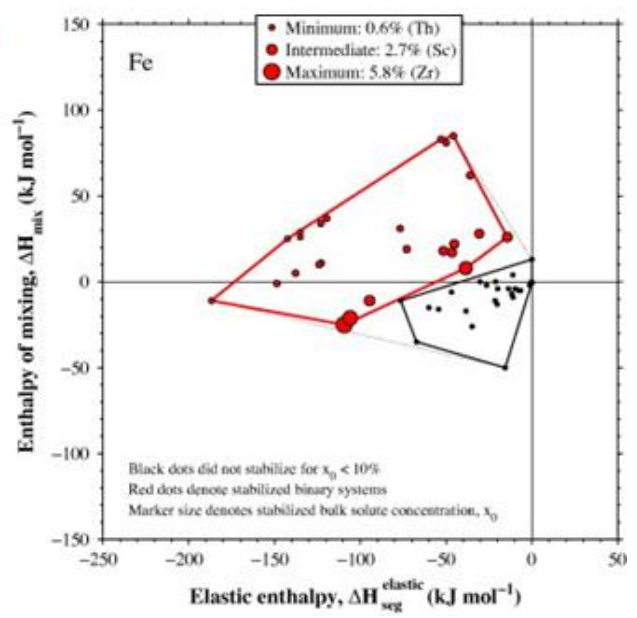

Fig. 2. (a) The Gibbs free energy surface of a nanocrystalline alloy for a single global dopant concentration value, where the minimum denotes a stable nanocrystalline state. (b) Nanocrystalline stability map for Febased alloys, showing that the solute additions labeled in red will act as stabilizing dopants. Part (a) was reprinted from [38] and Part (b) was reprinted from [40], with permission from Elsevier.

A common feature of the theories above is that all grain boundaries are treated the same. In other words, the anisotropy of grain boundary segregation is not accounted for. While this was likely a necessary simplification for early models, a number of important details are lost. Wynblatt and Chatain [41] formulated a detailed grain boundary segregation model that accounts for all five macroscopic parameters that determine a grain boundary's character. Although these authors showed that most regular solution models can be derived from this more complicated formulation with appropriate assumptions, they also showed that segregation can be sensitive to all five degrees of freedom. In addition, the existing thermodynamic models also treat interfaces as continuum features, without accounting for atomic structure. The details of grain boundary structure will be inherently important for stabilization, as the atomic structure sets the possible options for segregation sites.

Complexion theory is a useful tool for describing such segregation, as it offers an atomistic picture of local structural and chemical variations. In fact, the driving force for a complexion transition is a reduction in interfacial energy [42], directly fitting into a 
thermodynamic discussion of thermal stabilization. First and foremost, the local structural variations between different types of boundaries should impact segregation. The author's research group has been investigating complexion transitions using atomistic modeling techniques, with some previously unpublished results shown here. Hybrid Monte Carlo/molecular dynamics simulations were performed using the Large-scale Atomic/Molecular Massively Parallel Simulator (LAMMPS) code [43], with Monte Carlo providing equilibration of the chemical concentration within the sample while molecular dynamics allowed for local structural relaxations. All molecular dynamics simulations using a 1 fs integration time step. $\mathrm{Cu}-\mathrm{Zr}$ was chosen as a model alloy system with embedded-atom method potentials used to describe the $\mathrm{Cu}-\mathrm{Cu}$ and $\mathrm{Zr}-\mathrm{Zr}$ interactions, while a Finnis-Sinclair potential was used to describe the interactions between $\mathrm{Cu}$ and $\mathrm{Zr}$ atoms [44]. Each simulation cell contained between 200,000 and 300,000 atoms depending on the boundary type, and periodic boundary conditions were applied in all directions. The samples were first equilibrated with a conjugate gradient minimization technique and then a Nose-Hoover thermo/barostat was used to further relax the sample for 200 ps under zero pressure at different temperatures. Thereafter, doping with $\mathrm{Zr}$ atoms was simulated using a Monte Carlo method in a variance-constrained semi-grand canonical ensemble [45] after every 100 molecular dynamics steps, with the target global composition of $\mathrm{Zr}$ fixed to different values, in small increments. The chemical potential difference between $\mathrm{Zr}$ and $\mathrm{Cu}$ atoms was chosen to be $3.2 \mathrm{eV}$ to achieve relatively efficient convergence.

Here, we show results from three select interfaces which demonstrate the variety of segregation patterns that can be found. Fig. 3 presents $\Sigma 5$ (310), twist (111) $30^{\circ}$, and $\Sigma 11$ (311) boundaries, with atomic potential energy shown in (a) and dopant placement shown in (b). As 
dopants were added to the system and allowed to find their equilibrium position within the material, the $\mathrm{Zr}$ atoms segregated to the interfaces but with different patterns at different boundaries. The $\Sigma 5$ (310) boundary formed a single layer segregation pattern with $\mathrm{Zr}$ atoms decorating the tip of the repeating kite structure, while the twist (111) $30^{\circ}$ and $\Sigma 11$ (311) form bilayer and tri-layer structures. These three segregation patterns can be described as ordered complexions of Type I, III, and IV, respectively. Fig. 3(a) also demonstrates that although the segregation occurs at the interface, it is not always at the atomic site with the highest potential energy. This is most easily seen in the $\Sigma 5$ (310) boundary, where the preferred segregation sites sit between three high energy sites. As more $\mathrm{Zr}$ dopants were added to the system and/or temperature is increased, nanoscale amorphous intergranular films or wetting films eventually formed [46], likely because $\mathrm{Cu}-\mathrm{Zr}$ is a good binary glass forming alloy.
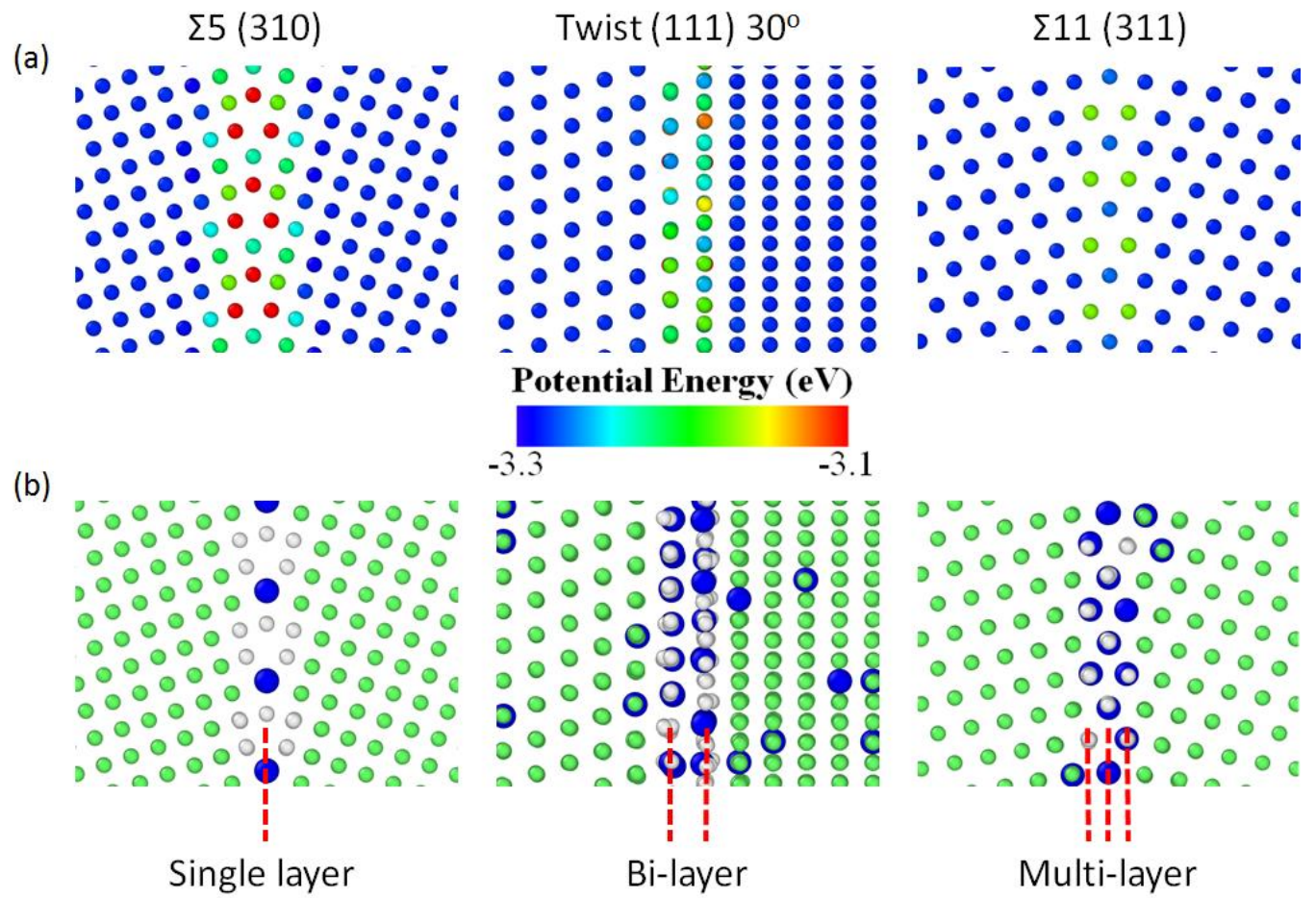

Fig. 3. (a) The local potential energy of three $\mathrm{Cu}$ grain boundaries, as well as (b) the resultant segregation patterns of $\mathrm{Zr}$ dopants after Monte Carlo/molecular dynamics simulations. In (b), crystalline and boundary $\mathrm{Cu}$ atoms appear green and white, respectively, while $\mathrm{Zr}$ atoms are blue. Different ordered complexions of Type I, III, and IV are found, depending on the boundary character. 
Even more subtle atomic aspects of grain boundary structure such as local free volume can affect segregation and complexion formation. Frolov et al. [47] showed that multiple complexions could be accessed even in elemental metals if atomic density was free to change. This was accomplished in their simulations through the addition of free surfaces which acted as either a source or sink for atoms so that the system can automatically adjust its local density in the boundary core. A first-order transition between multiple distinct Type II complexions was observed for both $\Sigma 5$ (310) and $\Sigma 5$ (210) boundaries when local density was allowed to vary. These complexions varied in their atomic structure, with the repeating unit switching between various kite-shaped structures. An example is shown in Fig. 4(a), where the normal kite structure of a $\Sigma 5$ (310) boundary can transform to a split-kite structure when in the presence of a free surface which allows variation in local density [47]. Such behavior is particularly relevant here because nanocrystalline metals often contain nonequilibrium grain boundaries with excess dislocations, misfit regions, or excess free volume [48-50]. These excess defects can be relaxed with annealing through a process call "grain boundary relaxation," giving another variable that can affect grain boundary structure and therefore segregation behavior. Frolov and coworkers [51] followed the paper discussed above with a study of Ag segregation to the different kite structures that are possible for a $\Sigma 5$ (310) interface, with two possible segregation states at $800 \mathrm{~K}$ shown in Fig. 4(b). If a normal kite structure is available, the Ag atoms segregate to the tip of the kite and form a single layer dopant pattern similar to the observation in Fig. 3(b) above. However, if the split-kite structure is available, a bi-layer segregation pattern occurs. It is important to note that the degree of site filling is not identical, as the single layer sites are all filled while the bi-layer sites are only partially filled. In any case, Frolov et al. found different 
relationships between the global and grain boundary composition depending on the kite structure present, again highlighting that boundary structure will influence the thermodynamics of segregation.

(a)

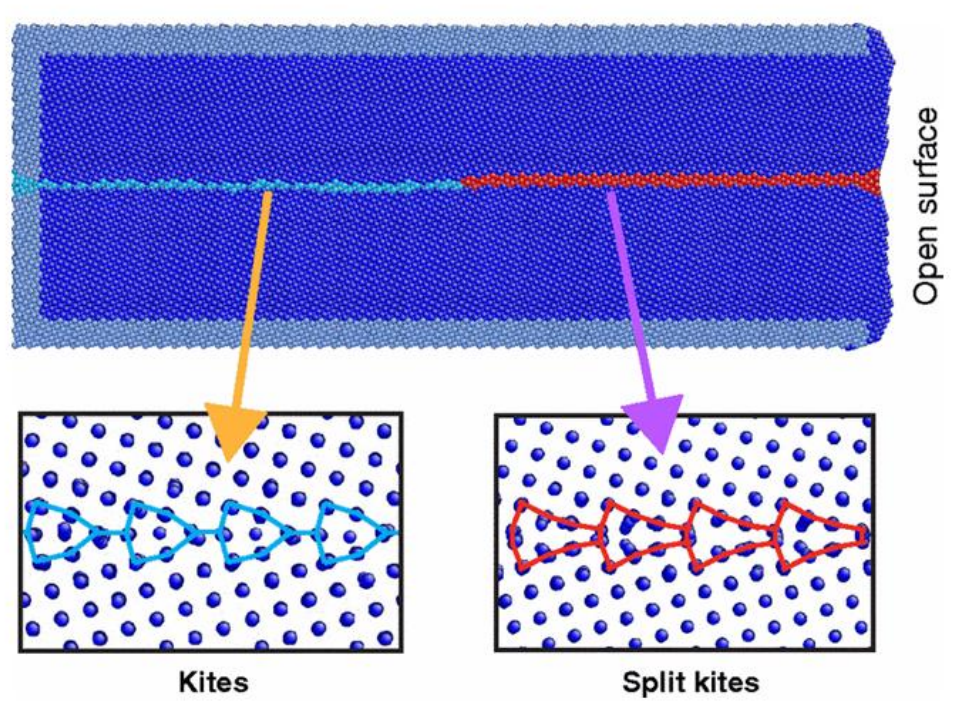

(b)

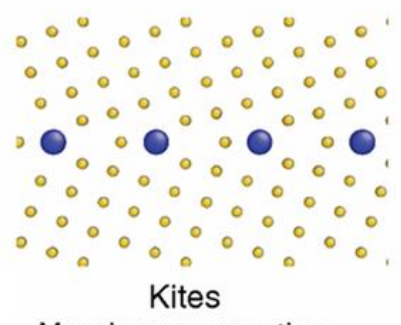

Monolayer segregation

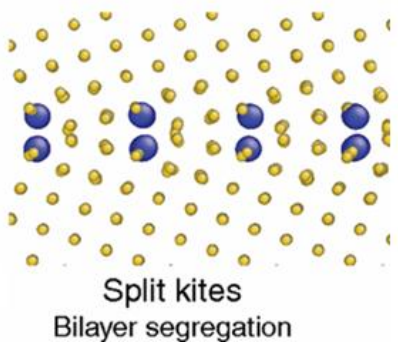

Fig. 4. (a) Normal kite structures at a $\Sigma 5(310)$ grain boundary transform to a split-kite configuration when atomic density is allowed to vary due to the presence of a free surface. (b) These different kite configurations experience different segregation patterns and dopant excess as a result of the variation in starting structure. Ag atoms appear blue in (b). Part (a) was reprinted from [47] with permission from Macmillan Publishers Ltd. Part (b) was reprinted with permission from [51], Copyright 2013 by the American Physical Society.

Both grain boundary character and other variables such as free volume should affect segregation patterns in nanocrystalline metals. The variation of complexion type show in Figs. 3 and 4 means that different amounts of dopant atoms would be needed to fill all segregation sites due to the difference in layer number. Even a single layer complexion can require a variety of dopant atoms per unit surface area, depending on the density of adsorption sites. The $\Sigma 5$ (310) boundary experienced single layer segregation but the spacing between dopants was set by the repeating structural unit of the grain boundary and would be different for another type of interface that experiences single layer segregation. For example, Nie et al. [52] also found single layer segregation of $\mathrm{Gd}$ and $\mathrm{Zn}$ atoms along coherent twin boundaries in $\mathrm{Mg}$, but with different dopant spacing. Variations in segregation patterns due to unrelaxed grain boundaries might also 
be an important feature to include in future models. Details like this are missed by current thermodynamic theories and would alter the global concentration needed to stabilize a nanocrystalline metal. The incorporation of different complexion states as possible segregation patterns, as well as a further understanding of the distribution of complexions types expected in random grain boundary network would further the field's understanding of segregation-induced thermal stability.

Type V and VI complexions can also be important boundary structures for nanocrystalline thermal stability, as these are often the type of complexions that will form at high temperatures. Luo and Shi [53] developed a theoretical model based of the Miedema model and computational thermodynamics to describe the formation of nanoscale amorphous intergranular films (Type V complexions) in binary alloys. This model predicted that disordering of grain boundary structure would occur at temperatures as low as $60-85 \%$ of the solidus temperature for the alloy. In this paper and later work [20], these authors were able to create "grain boundary phase diagrams" that show where such complexions are overlaid onto bulk phase diagrams. Khalajhedayati and Rupert [54] observed the formation of structurally disordered complexions when studying the thermal stability of nanocrystalline $\mathrm{Cu}-3$ at.\% $\mathrm{Zr}$ alloys fabricated by ball milling. These authors found nanoscale amorphous intergranular films after annealing at $850{ }^{\circ} \mathrm{C}$ $\left(0.9 T_{\text {melting }}\right)$ and $950{ }^{\circ} \mathrm{C}\left(0.98 T_{\text {melting }}\right)$ for $1 \mathrm{~h}$ followed by rapid quenching, while only ordered interfaces were observed after annealing at temperatures of $750{ }^{\circ} \mathrm{C}$ and below. Amorphous intergranular film thickness was found to increase with annealing temperature, fitting the theoretical predictions of Luo and coworkers $[20,29,53]$ that higher temperatures promote thicker films. Overall, amorphous complexions with thicknesses ranging from $0.5 \mathrm{~nm}$ to $5.7 \mathrm{~nm}$ were observed by Khalajhedayati and Rupert [54], with an approximately $5 \mathrm{~nm}$ thick example shown 
in Fig. 5. These authors also found that the $\mathrm{Zr}$-doped nanocrystalline $\mathrm{Cu}$ was extremely stable against grain growth, only coarsening to an average grain size of $54 \mathrm{~nm}$ after spending 1 week at $950{ }^{\circ} \mathrm{C}$. While the microstructure also included small $\mathrm{ZrC}$ particles that provided some kinetic contribution, thermodynamic effects from complexion formation was found to be a major contributor to this stability.

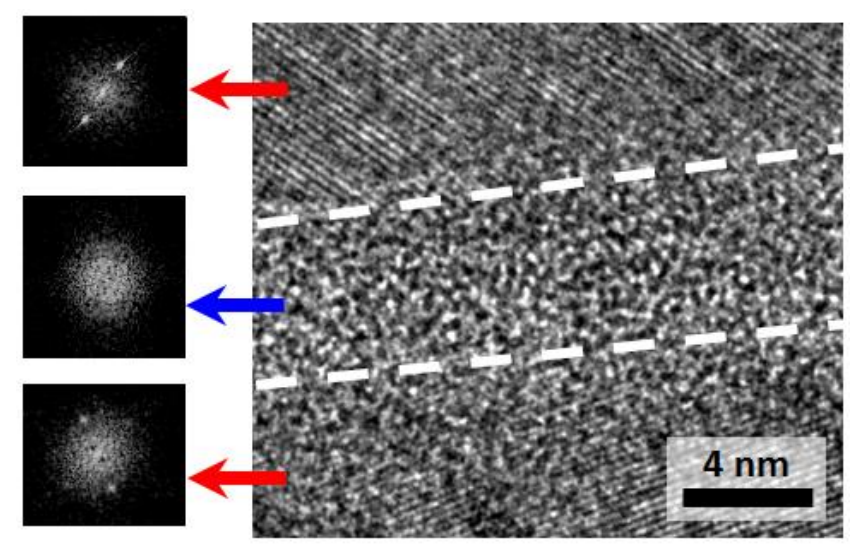

Fig. 5. High resolution TEM image of a grain boundary in nanocrystalline $\mathrm{Cu}-3$ at.\% $\mathrm{Zr}$, where an amorphous intergranular film of $5 \mathbf{~ n m}$ thickness is shown. Fast Fourier transform patterns highlight that the interfacial film exhibits a disordered structure.

In addition to reducing boundary energy and stabilizing grain size, segregation and complexion transitions can be important for nanocrystalline metals due to their impact on mass transport properties such as diffusion rates. Recall that a large reason thermal stability is important is that nanostructured materials created by bottom up processes must be consolidated into larger pieces, meaning rapid mass transport can be beneficial in this case. Measurements of grain boundary diffusivity in $\mathrm{Cu}-\mathrm{Bi}$ alloys showed an abrupt increase of two orders of magnitude in the diffusivity at relatively low Bi concentrations [25], which was connected to a discontinuity in the slope of the grain boundary energy [55] (i.e., a signal that a complexion transition occurred). Complexion-induced diffusivity transitions were also found observed in Ni-Bi and $\mathrm{Cu}-\mathrm{Bi}$ by Tai et al. [56] using secondary ion mass spectroscopy depth profiling. If grain boundary energy is reduced due to a reduction in enthalpy, one would expect a reduction in 
diffusivity [57]. Thus, the increase in diffusivity with a transition to a lower energy state signifies that entropy changes may be more important [56]. In any case, accelerated mass transport due to complexion formation at heavily doped boundaries should speed up consolidation or allow consolidation to occur at lower temperatures. However, there is also the potential for deleterious thermodynamic effects concerning consolidation. If a reduction in surface energy mimicking the reduction in grain boundary energy occurs with doping, the driving force for densification will also be reduced. A more complete picture of these competing thermodynamic and kinetic considerations that occur during the consolidation of nanocrystalline materials is needed.

A major challenge concerning the stabilization of nanocrystalline materials, especially concerning the discussion of the importance of complexions above, is the characterization of segregation state and complexions with high fidelity throughout a material. If boundaries form different ordered complexions and go through premelting or disordering transitions at different times, one would expect a distribution of complexion types to be found throughout a random nanocrystalline grain boundary network. This goal is difficult to achieve because it requires (1) atomic resolution to distinguish between ordered and disordered complexions, (2) local composition information to look for chemical ordering or distinguished between doped and undoped boundaries, and (3) the characterization of large numbers of boundaries to obtain statistical data about complexion type or segregation level throughout the material.

Unfortunately, there is no one characterization tool that can achieve all three requirements. TEM has excellent spatial resolution, making it the best tool for investigating the atomic structure of boundaries, and can also provide atomic-scale chemical information when combined with detection methods such as electron energy loss spectroscopy (EELS) [58] or 
energy-dispersive X-ray spectroscopy (EDS) [59]. Large numbers of boundaries can also be investigated with TEM, since specimens typically have electron transparent regions that have inplane dimensions of microns ( 100-1000 times larger than nanocrystalline grain sizes), meaning a single TEM sample contains tens of thousands of interfaces. However, a boundary must be in an edge-on condition, or parallel to the electron beam, to be studied with high resolution TEM. In addition, atomic-scale resolution requires lattice fringes in both grains to be visible, so high resolution TEM studies are often limited to tilt boundaries. Since a random polycrystal is a three-dimensional network of interfaces, it might not be able to achieve these condition for connected boundaries, meaning that most observations will be one off measurements of boundary structure and network features such as the connectivity of complexion types cannot be studied directly with TEM.

On the other hand, atom probe tomography (APT) can obtain three dimensional compositional data, atom-by-atom, making it excellent for measuring chemical segregation patterns throughout a volume. Unfortunately, APT has limited spatial resolution due to trajectory aberrations, thermal agitation of surface atoms, and local magnification effects [60], meaning that atomic resolution is not possible. The best that can be done is to identify a few crystallographic plane spacings [61,62], which are inherently larger than the atomic spacing. In addition, APT is only able to probe very small volumes of materials, limiting the number of boundaries that can be investigated. A typical APT sample is approximately $100 \mathrm{~nm}$ long and 50 $\mathrm{nm}$ wide. Even for a nanocrystalline material, this means that we would only expect to find a small number of grain boundaries in a given sample. To obtain a complete description of the variety of boundaries contained within a doped polycrystal, one would need to look at dozens of APT samples. 
A promising path forward might involve the combination of these two techniques. Herbig et al. [63] combined APT with TEM to study boundary segregation of $\mathrm{C}$ in a steel. Nanobeam diffraction was used to measure grain orientations at each point, then the sample was field evaporated to obtain chemical composition throughout the sample. Fig. 6 shows a twodimensional projection of the three-dimensional APT data projected onto a scanning TEM image of the grain structure. Herbig et al. then calculated the Gibbs interfacial excess of $\mathrm{C}$ in atoms $/ \mathrm{nm}^{2}$ for 121 boundaries investigated in seven different samples from the same material, plotting this information against misorientation angle to look for trends. The volume probed is still a limitation with this TEM/APT technique and very few boundaries per sample can be studied if grain size gets even slightly larger, but the combination of atomic-scale structural and chemical resolution is promising. Another alternative for studying networks of complexions would be to simulate the segregation behavior of polycrystalline materials with large numbers of grains, perhaps through Monte Carlo/molecular dynamics simulations similar to the bicrystal examples shown earlier. Atomistic simulations are routinely able to model hundreds of nanograins [64], meaning thousands of interfaces could be studied with no inherent limitations on resolution. Analysis techniques have been developed to extract information such as crystal orientation [65], with data from the two crystals across the boundary giving misorientation, and grain boundary normal [66], meaning it is possible to have all five macroscopic degrees of freedom for a boundary in addition to an image of its atomic structure and segregation state from atomistic modeling. 


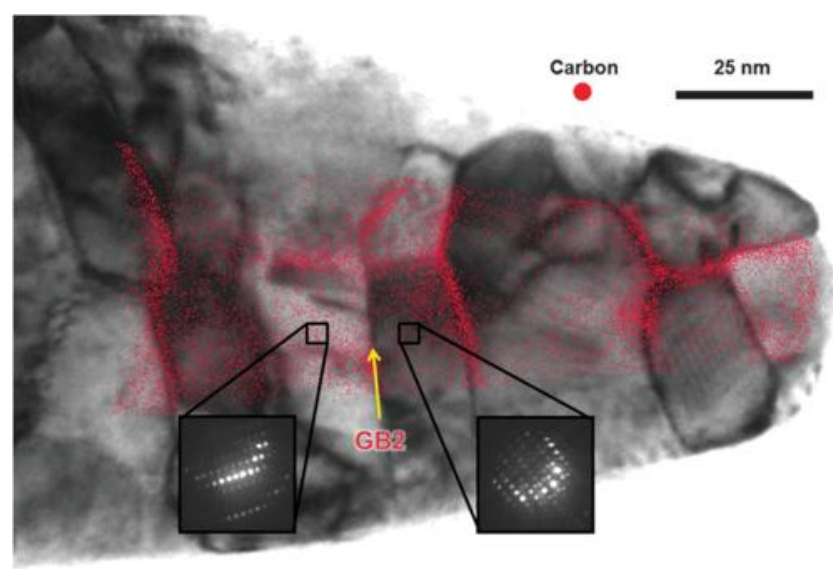

Fig. 6. Two-dimensional projection of APT data from a ferrite steel sample overlaid on top of a scanning TEM micrograph. TEM can provide crystallographic information or structural information about the interfaces, while APT gives detailed segregation maps. Reprinted with permission from [63], Copyright 2014 by the American Physical Society.

\section{Mechanical Behavior of Nano-grains}

A compilation of yield strength and strain-to-failure data from $\mathrm{Cu}$ and $\mathrm{Cu}$-based alloys is presented in Fig. 7, where a trade-off between these two properties is obvious. All of the data from both microcrystalline and nanocrystalline $\mathrm{Cu}$ falls within the grey envelope that denotes this trend. Most strategies for avoiding the strength-ductility dilemma in nanocrystalline metals are based upon either incorporating a variety of grain sizes into the microstructure to make a type of composite material or adding twin boundaries. One example of the first strategy is the use of a bimodal grain structure, comprised of occasional micron-sized grains embedded into a nanocrystalline matrix $[2,67]$. Another is the recent development of gradient nanostructured materials, where grain size changes smoothly from the nanometer scale on the surface of a component to the micron scale in the interior $[68,69]$. This type of microstructure has been shown to induce a macroscopic strain gradient throughout the material, resulting in extra strain hardening and delay failure [70]. Nanotwinned metals are typically comprised of grains with diameters of 500-1000 nm, which contain twin boundaries with average spacings of 5-100 nm 
[71,72]. This unique microstructure allows for both soft and hard modes of deformation [73], with slip parallel and perpendicular to the twin boundaries, respectively. A few representative examples are plotted in Fig. 7 for bimodal grain size and nanotwinned $\mathrm{Cu}$. A better combination of strength and ductility can be achieved compared to materials with a single grain size, yet these strategies do not change the fundamental issue that one property comes at the expense of the other (see the nanotwinned $\mathrm{Cu}$ data in Fig. 7, for example). To find a way to increase ductility while maintaining or even increasing strength, structural features beyond grain size or twin boundary density must be explored.

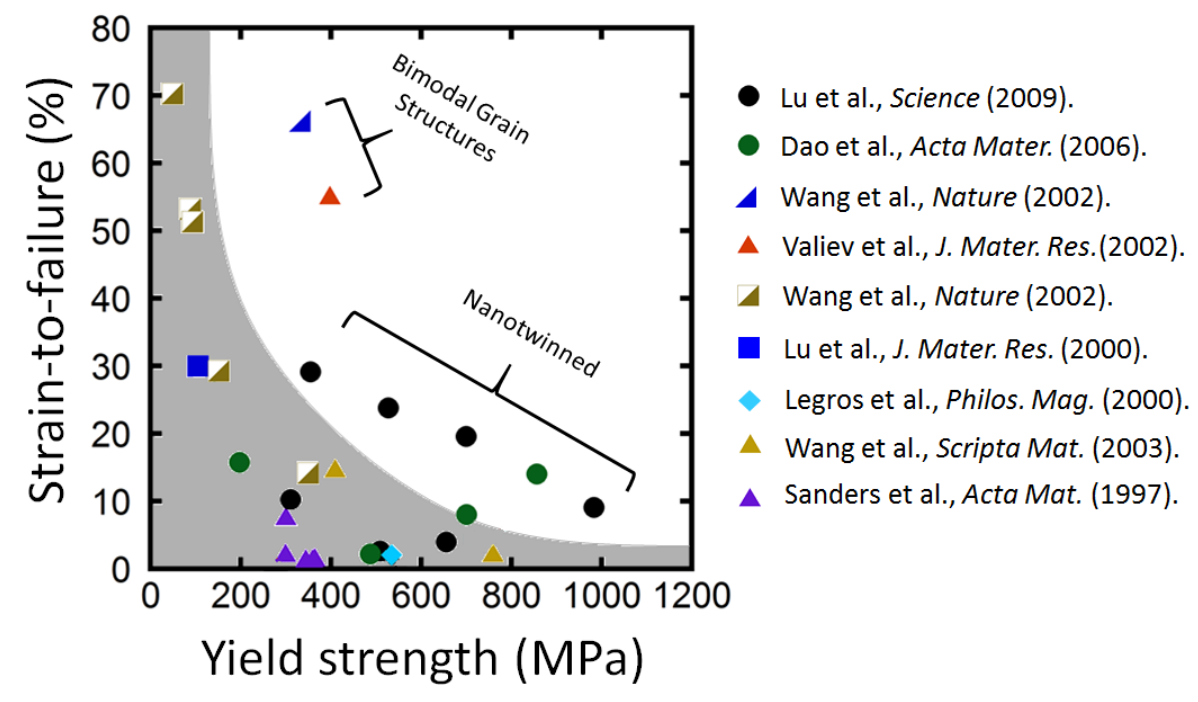

Fig. 7. Strain-to-failure and yield strength data for microcrystalline and nanocrystalline $\mathrm{Cu}$ and $\mathrm{Cu}$-based alloys. A general trend of either high strength but brittle failure or low strength and good ductility is observed. Advanced materials such as bimodal grain sized $\mathrm{Cu}$ and nanotwinned $\mathrm{Cu}$ can push outside the grey envelope, but strength still comes at the expense of ductility. Literature data is taken from [2$4,67,71,73,74]$.

To develop unique material design strategies, it is instructive to explore the relevant physical mechanisms behind plastic deformation and premature failure in nanocrystalline metals. For grain sizes between 10 and $100 \mathrm{~nm}$, dislocations still carry plastic deformation but intergranular dislocation networks do not form. Plasticity occurs by a process of dislocation nucleation, propagation through the nano-grain with periodic pinning at the grain boundaries, 
and finally dislocation absorption into the opposite interface $[75,76]$. The nucleation and pinning events have been used to create robust models that describe how grain size $[77,78]$ and solid solution concentration [79,80] affect nanocrystalline strength. Bitzek et al. [81] isolated the dislocation absorption event by artificially adding a full dislocation glide loop to a nanocrystalline molecular dynamics model, then applying a stress so that the loop expanded and interacted with the grain boundary. Detailed analysis after the absorption event showed that, although the dislocation has disappeared and the grain looked perfect at first glance, important changes had occurred on the atomic scale. This is show in Fig. 8(a), where the images in the left and right columns shown the local hydrostatic pressure and local resolved shear stress before and after dislocation absorption, respectively. Although the dislocation is completely absorbed, the grain boundary has multiple sites with high local stresses. Since multiple absorption events are likely to occur in a given grain, these should be potential failure sites. Pan and Rupert [82] tested this hypothesis by simulating repetitive dislocation absorption using MD simulation, finding that atomic shuffling within the boundary is required to accommodate the incoming plastic strain brought by each dislocation. When this process is inefficient, cracking commences. Once a crack has nucleated, the high density of grain boundaries can act as an easy path for crack propagation. Farkas et al. [83] showed that cracks travel along intergranular paths in a nanocrystalline grain structure, as shown in Fig. 8(b), first creating nano-voids at boundary sites ahead of the crack which then coalesce to grow the main fracture surface. Therefore, crack nucleation from inefficient dislocation nucleation and intergranular crack propagation are identified as the two important physical phenomena controlling nanocrystalline fracture. 
(a)
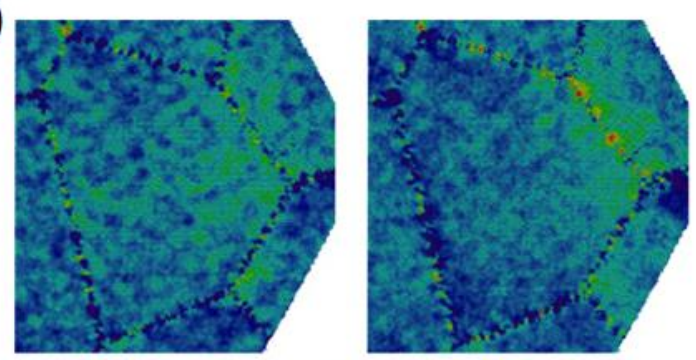

$-2 \mathrm{GPa}$

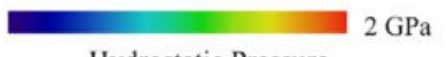

Hydrostatic Pressure
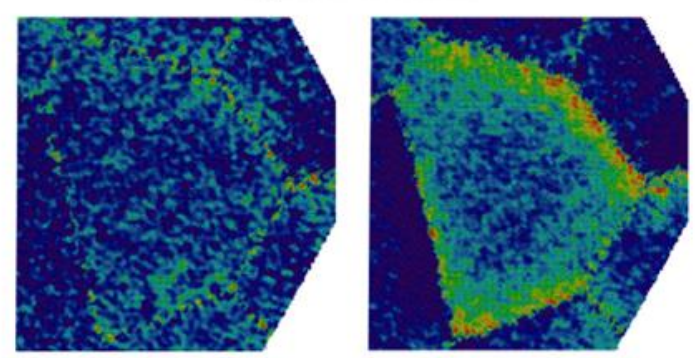

$-1 \mathrm{GPa}$

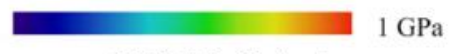

$\tau(B C(d))$ in Grain 1 (b)
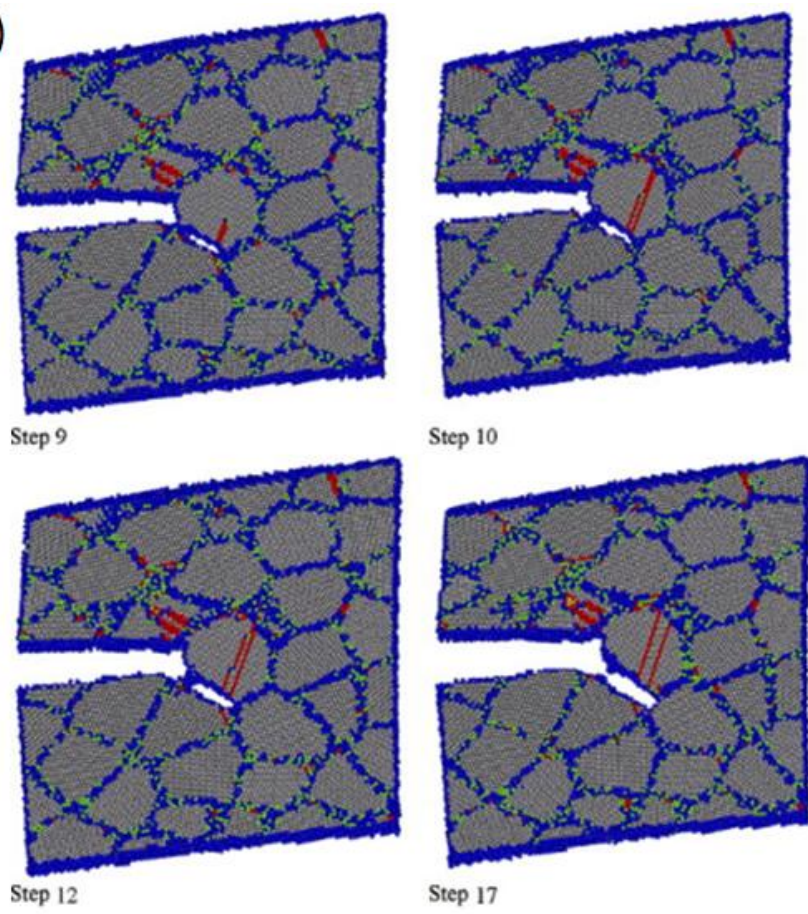

Step 10

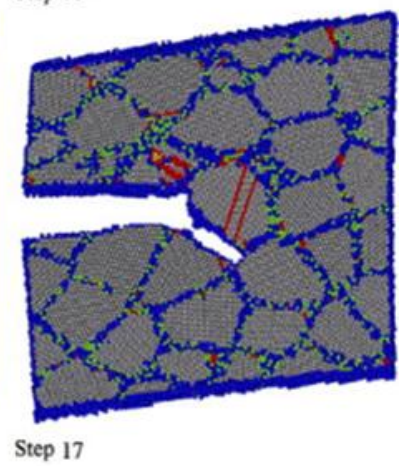

Fig. 8. (a) Nanocrystalline grain boundaries before (left) and after (right) a dislocation loop was absorbed into the boundaries during a molecular dynamics simulation. Sites of both high hydrostatic pressure (top) and high resolved shear stress (bottom) are found. (b) A molecular statics model of nanocrystalline Ni with a crack that travels along an intergranular path during progressive loading. Part (a) reprinted with permission from [81] @ IOP Publishing, all rights reserved. Part (b) reprinted from [83], with permission from Elsevier.

The two mechanisms above have a commonality that activity at the grain boundary is

critical. A reasonable hypothesis is then that grain boundary structure should be important for controlling failure. To test this idea, Khalajhedyati and Rupert [84] probed the uniaxial compressive deformation of electrodeposited nanocrystalline $\mathrm{Ni}-\mathrm{W}$ in both the as-deposited state, where the boundaries are unrelaxed and have additional disorder (see discussion in Section 3 above), as well as the relaxed state, where more ordered grain boundary structures are found. For materials with $d=15 \mathrm{~nm}$ having nonequilibrium grain boundaries, micropillars could be compressed to true strains of 10-20\% before sudden failure occurred, as shown in Fig. 9(a). Fig. 9(b) demonstrates that relaxation of the interfaces in this same grain size material dramatically reduced the compressive strain-to-failure to $\sim 5 \%$. These results highlight the importance of 
grain boundary state for nanocrystalline failure, with added structural disorder isolated as a positive feature for improving failure resistance.

(a)

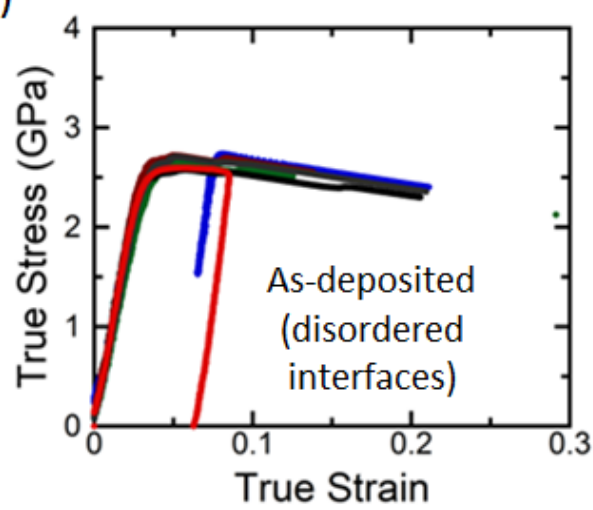

(b)

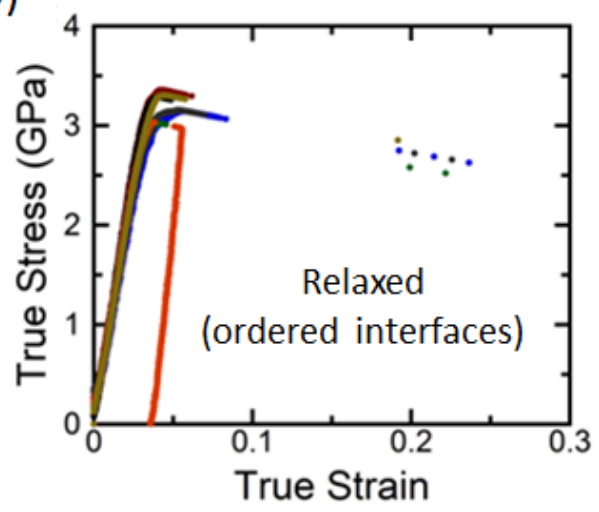

Fig. 9. Micropillar compression stress-strain curves for nanocrystalline Ni-W with $d=15 \mathrm{~nm}$ (a) when grain boundaries are in a nonequilibrium state or disordered and (b) when boundaries are relaxed or ordered. Structural disorder at nanocrystalline grain boundaries is beneficial for increasing the failure resistance. Reprinted from [84], with permission from Elsevier.

Complexions offer a way to tailor the level of structural order or disorder at a grain boundary in a controlled manner. As discussed in Section 2, complexion Types I, III, and IV represent additional structural order over an undoped grain boundary, while complexion Types $\mathrm{V}$ and VI are often disordered. Liquid metal embrittlement is a phenomenon where metals which are usually ductile become prone to brittle intergranular failure after exposure to certain liquid metals. Luo et al. [85] studied this phenomenon is Ni-Bi using aberration-corrected TEM, finding that this embrittlement was due to the formation of bi-layer complexions from preferential Bi adsorption. These author suggested that the Ni-Bi bonds were much stronger than the $\mathrm{Bi}-\mathrm{Bi}$ bonds, leading to incoherent and weakly bonded $\mathrm{Bi}-\mathrm{Bi}$ bonds across the interface. Sigle et al. [86] made similar observations in Al-Ga, another well-known system that undergoes liquid metal embrittlement, finding multi-layer segregation of $\mathrm{Ga}$ with a thickness of three monolayers at a $\Sigma 11$ [110] (311) grain boundary. Again, the embrittlement could be connected to the fact that $\mathrm{Ga}$ atoms form stronger bonds to $\mathrm{Al}$ atoms than to other $\mathrm{Ga}$ atoms. On the other 
hand, amorphous $\mathrm{C}$ interfacial films have been shown to improve the fracture toughness of nanostructured $\mathrm{B}_{4} \mathrm{C}$, serving as soft interfaces which can promote grain boundary sliding [87]. Although these examples do not concern nanocrystalline metals, they support the idea that highly ordered grain boundaries are brittle while disordered complexions can be beneficial for increasing failure resistance.

Pan and Rupert [88] used molecular dynamics to simulate how disordered boundaries react to repetitive dislocation absorption. An artificial dislocation source was operated at the center of a $\mathrm{Cu}$ bicrystal sample while clean interfaces and $\mathrm{Cu}-\mathrm{Zr}$ amorphous intergranular films with a variety of thicknesses, created by doping and melting a small region, were added along the grain boundaries. This simulation cell is presented in Fig. 10(a), where radial distribution functions (RDFs) of the local structure show that the boundary is amorphous with some limited short range order. Fig. 10(b) shows the atomic model for an amorphous film of $5.7 \mathrm{~nm}$ thickness where atoms are colored according to their atomic von Mises equivalent shear strain, with higher applied macroscopic strains on the simulation cell meaning that progressively more absorption events have occurred. It is clear from this figure that the plastic strain brought by the incoming dislocation is diffused into a large volume in the amorphous interface. Further analysis showed that this strain sharing behavior delayed crack nucleation significantly, with thicker disordered grain boundaries being able to absorb more dislocations. In fact, the amorphous intergranular film with $5.7 \mathrm{~nm}$ thickness was able to absorb 3.5 times as many dislocations as a clean interface. In addition, the diffuse nature of the atomic strain reduced the local hydrostatic stress at the interface, which then slowed crack propagation as well. The results shown in Fig 10 are important because they simulate the key physical events and structural features that a nanocrystalline metallic alloy with boundaries decorated by amorphous complexions (Type V or 
VI), would experience. This is effectively what was created in [54], suggesting that such alloys should be much more ductile than nanocrystalline $\mathrm{Cu}$ usually is. Amorphous-crystalline nanolaminates fabricated by Wang et al. [89] support such a conclusion as well. Although these authors added amorphous layers by co-sputtering and solid state amorphization, a different physical process, they found that the addition of an amorphous layer between layers of metallic nano-grains increased ductility. Brandl et al. [90] used atomistic techniques to isolate the effect of the amorphous-crystalline interface, finding that the elastically softer amorphous layer can actually attract dislocations to further promote absorption. Unfortunately, nanolaminates only added disordered interfacial features in one direction within the film (in-plane due to the use of a deposition technique), creating a very anisotropic material. A random polycrystal decorated with amorphous complexions should have even better ductility. Khalajhedayati et al. [91] recently provided evidence of this effect, showing that $\mathrm{Cu}-\mathrm{Zr}$ alloys with amorphous intergranular films were an order of magnitude more ductile than pure nanocrystalline $\mathrm{Cu}$.
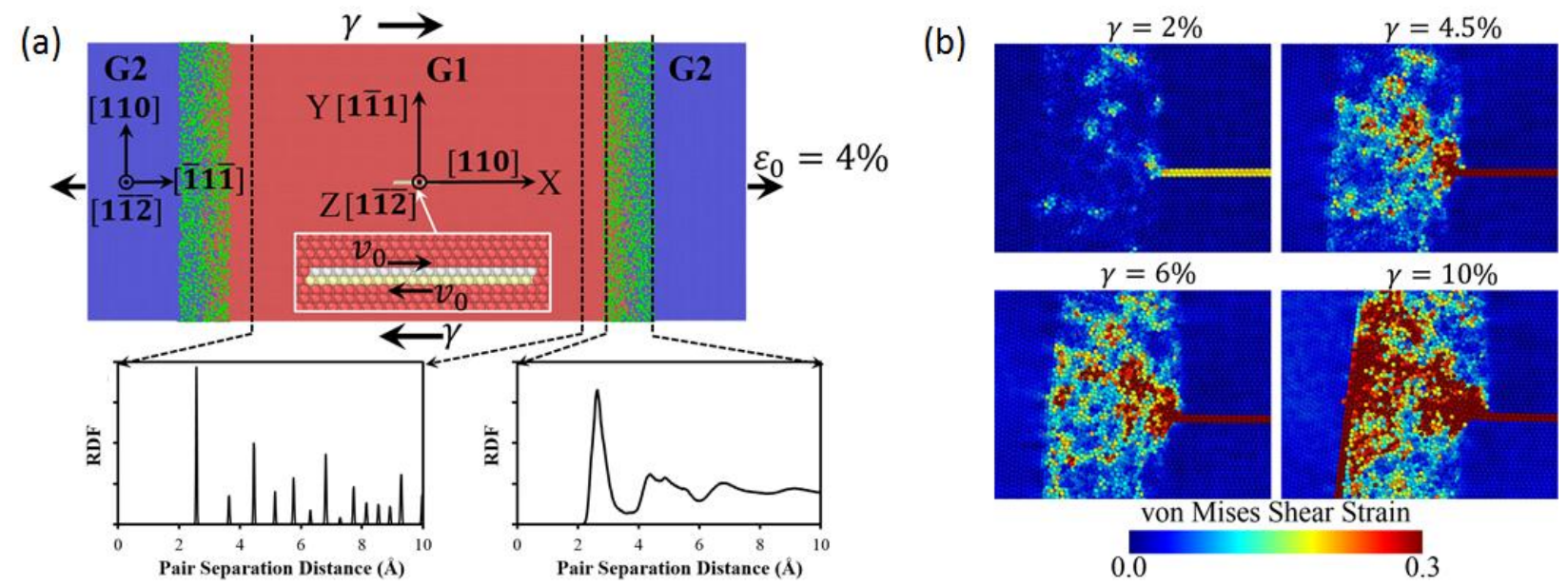

Fig. 10. (a) A bicrystal sample with amorphous intergranular films containing a dislocation source in the center. Such a simulation can be used to simulate the repeated dislocation absorption that lead to failure in nanocrystalline metals. (b) Atomic distribution of von Mises shear strain in a sample with a $5.7 \mathrm{~nm}$ thick amorphous intergranular film. The disordered interface accommodates the incoming dislocations into a large region, delaying both crack nucleation and propagation. Reprinted from [88], with permission from Elsevier. 
The discussion above shows that disorder can be beneficial, but a number of other factors such as bond type and short-range order may modify such behavior or even negate any positive effect. Amorphization due to $\mathrm{S}$ at grain boundaries in $\mathrm{Ni}$ is a well-known case of segregationinduced embrittlement [92]. Molecular dynamics have shown that this amorphization reduces the shear strength of grain boundaries in Ni by an order of magnitude [93]. Similar glassy films in W-Ni were also found to embrittle [94], suggesting that not all amorphous intergranular films are created equal. Specifically, chemical bonding type may be an essential factor. Messmer and Briant [95] showed that embrittling dopants in general remove charge from the metal-metal bonds which hold the interface together. More recently, Gibson and Schuh [96] developed a model for describing how cohesive energy of a boundary is altered by equilibrium segregation. This model was shown to capture well-known literature data, with embrittling agents in $\mathrm{Fe}$ and $\mathrm{Au}$ generally being found to be non-metals or metalloids. As a whole, this suggest that retaining metallic bond character, perhaps by restricting the choice of elements to transition metal that segregate, in disordered complexions is essential for improving ductility. It is also unclear how local structural measures such as short-range order will affect dislocation absorption and the ductility of nanocrystalline metals. Such details are known to be very important for plasticity in metallic glasses [97], so it stands to reason that they will also have a noticeable effect here.

Finally, the fraction of boundaries that have a certain complexion structure, as well as the connectivity of this complexion network throughout the material, is likely important for understanding how to improve the ductility of nanostructured metals. An amorphous metal is a brittle phase on its own [98], so the positive effects it can bring are predicated on not letting the failure of this complexion itself control the overall behavior. We said earlier that thicker amorphous films can absorb more dislocations, but if thick disordered films formed an 
interconnecting network along all grain boundaries and became the majority phase, this may begin to converge toward the expected metallic glass response. The work of Zhang et al. [99] on $\mathrm{Cu}-\mathrm{Zr}$ films which underwent solid-state amorphization supports such a hypothesis. Although the amorphous phase in these films was not an equilibrium complexion but rather a metastable state and grain size was not held constant, these authors found that a continuous amorphous network in the composite films lead to a decrease in tensile ductility. There is likely a balance to be made, where thick films are desired but scattered throughout the grain boundary network to maximize ductility.

The addition of certain complexions to a nanocrystalline metal should also be beneficial for strength. The dislocation nucleation and pinning events which control strength also depend on grain boundary structure, with a number of studies showing that lowering the grain boundary energy increases strength. Vo et al. [100] investigated the yield strength of dilute nanocrystalline $\mathrm{Cu}-\mathrm{Nb}, \mathrm{Cu}-\mathrm{Ag}$, and $\mathrm{Cu}-\mathrm{Fe}$ alloys with $d=8 \mathrm{~nm}$ using atomistic simulations. Monte Carlo/molecular dynamics was used to simulate the doping process, in a manner similar to the examples described in Section 3 above, followed by molecular dynamics to test mechanical behavior. Yield strength was found to increase monotonically with reductions in grain boundary energy, reaching values near the theoretical strength for $\mathrm{Cu}$. Ozerinc et al. [101] showed a similar strengthening effect in nanocrystalline thin films of $\mathrm{Cu}-\mathrm{Nb}$ and $\mathrm{Cu}-\mathrm{Fe}$ to provide experimental validation for the connection between grain boundary energy and strength. Segregation of $\mathrm{C}$ to interfaces and a reduction of grain boundary energy was found to be critical in the work of Li et al. [102], who fabricated bulk nanocrystalline steel with strength near the theoretical limit. While the studies above focused on ordered grain boundaries, the recent work of Khalajhedayati and Rupert [54] demonstrated that both ordered and disordered complexions in 
nanocrystalline $\mathrm{Cu}-\mathrm{Zr}$ can increase strength, with disordered boundaries actually having the larger strengthening effect. Therefore, the unique opportunity offered by complexions is that they can potentially be used to improve both strength and ductility of nanocrystalline metals while prior material design strategies always lead to a trade-off between these properties.

\section{Summary and Final Remarks}

The lack of thermal stability and limited ductility in nanocrystalline metals can perhaps be treated if the chemical composition and atomic structure of boundaries could be tuned by a material's designer. Complexions offer a controlled way of doing just this in order to reduce excess grain boundary energy and enable grain boundaries to repeatedly absorb dislocations. The detailed descriptions of interfacial states, including different segregation patterns, boundary thicknesses, and measures of structural and chemical order/disorder on the atomic scale, provided by complexion theory can improve the current state-of-the-art techniques for addressing these limiting issues if they can be adequately integrated into current theories. In this work, we have highlighted a number of connections between nanocrystalline metals and complexions, as well as identified challenging tasks in the areas of characterization, simulation, and theory of nano-grained microstructures. If the two persistent issues discussed here can be solved by innovation in the area of boundary design so that bulk pieces can be made and a synergy between strength and ductility is achieve, if is quite possible that nanocrystalline metals will be the material of choice for structural applications for the foreseeable future.

\section{Acknowledgements}


This work was supported by the U.S. Department of Energy, Office of Science, Basic Energy Sciences, under Award No. DE-SC0014232. T.J.R. thanks Dr. Zhiliang Pan for providing the simulations of ordered complexions at different $\mathrm{Cu}-\mathrm{Zr}$ grain boundaries shown in Section 3. 


\section{References}

1. Palumbo, G., Thorpe, S. J. and Aust, K. T. "On the Contribution of Triple Junctions to the Structure and Properties of Nanocrystalline Materials." Scripta Metallurgica Et Materialia 24, 1347-1350, (1990).

2. Wang, Y., Chen, M., Zhou, F. and Ma, E. "High tensile ductility in a nanostructured metal." Nature 419, 912-915, (2002).

3. Legros, M., Elliott, B. R., Rittner, M. N., Weertman, J. R. and Hemker, K. J. "Microsample tensile testing of nanocrystalline metals." Philos. Mag. A 80, 1017-1026, (2000).

4. Wang, Y. M. et al. "Microsample tensile testing of nanocrystalline copper." Scr. Mater. 48, 1581-1586, (2003).

5. Maung, K., Earthman, J. C. and Mohamed, F. A. "Inverse Hall-Petch behavior in diamantane stabilized bulk nanocrystalline aluminum." Acta Mater. 60, 5850-5857, (2012).

6. Rupert, T. J., Cai, W. and Schuh, C. A. "Abrasive wear response of nanocrystalline Ni-W alloys across the Hall-Petch breakdown." Wear 298-299, 120-126, (2013).

7. Padilla, H. A., II, Boyce, B. L., Battaile, C. C. and Prasad, S. V. "Frictional performance and near-surface evolution of nanocrystalline Ni-Fe as governed by contact stress and sliding velocity." Wear 297, 860-871, (2013).

8. Boyce, B. L. and Padilla, H. A., II. "Anomalous Fatigue Behavior and Fatigue-Induced Grain Growth in Nanocrystalline Nickel Alloys." Metallurgical and Materials Transactions A 42, 1793-1804, (2011).

9. Natter, H., Schmelzer, M. and Hempelmann, R. "Nanocrystalline nickel and nickelcopper alloys: Synthesis, characterization, and thermal stability." J. Mater. Res. 13, 11861197, (1998).

10. Eckert, J., Holzer, J. C., Krill, C. E. and Johnson, W. L. "Structural and thermodynamic properties of nanocrystalline fcc metals prepared by mechanical attrition." J. Mater. Res. 7, 1751-1761, (1992).

11. Gertsman, V. Y. and Birringer, R. "On the Room-Temperature Grain-Growth in Nanocrystalline Copper." Scripta Metallurgica Et Materialia 30, 577-581, (1994).

12. Tschopp, M. A., Murdoch, H. A., Kecskes, L. J. and Darling, K. A. "“Bulk" Nanocrystalline Metals: Review of the Current State of the Art and Future Opportunities for Copper and Copper Alloys." JOM 66, 1000-1019, (2014).

13. Ma, E. "Instabilities and ductility of nanocrystalline and ultrafine-grained metals." Scr. Mater. 49, 663-668, (2003).

14. Gianola, D. S. et al. "Stress-assisted discontinuous grain growth and its effect on the deformation behavior of nanocrystalline aluminum thin films." Acta Mater. 54, 22532263, (2006).

15. Sharon, J. A., Padilla, H. A. I. and Boyce, B. L. "Interpreting the ductility of nanocrystalline metals." J. Mater. Res. 28, 1539-1552, (2013).

16. Hart, E. W. "2-Dimensional Phase Transformation in Grain Boundaries." Scripta Metallurgica 2, 179, (1968).

17. Cahn, J. W. "Transitions and Phase-Equilibria Among Grain-Boundary Structures." Journal De Physique 43, 199-213, (1982). 
18. Sutton, A. P. and Balluffi, R. W. Interfaces in crystalline materials. (Clarendon Press; Oxford University Press, 1995).

19. Dillon, S. J., Tang, M., Carter, W. C. and Harmer, M. P. "Complexion: A new concept for kinetic engineering in materials science." Acta Mater. 55, 6208-6218, (2007).

20. Shi, X. M. and Luo, J. "Developing grain boundary diagrams as a materials science tool: A case study of nickel-doped molybdenum." Phys. Rev. B 84, 014105, (2011).

21. Dillon, S. J., Harmer, M. P. and Luo, J. "Grain Boundary Complexions in Ceramics and Metals: An Overview." JOM 61, 38-44, (2009).

22. Cantwell, P. R. et al. "Grain boundary complexions." Acta Mater. 62, 1-48, (2014).

23. Faraday, M. "Note on Regelation." Proceedings of the Royal Society of London 10, 440450, (1860).

24. Hsieh, T. E. and Balluffi, R. W. "Experimental study of grain boundary melting in aluminum." Acta Metall. 37, 1637-1644, (1989).

25. Divinski, S. et al. "Grain-boundary melting phase transition in the $\mathrm{Cu}-\mathrm{Bi}$ system." Phys. Rev. B 71, 104104, (2005).

26. Tang, M., Carter, W. C. and Cannon, R. M. "Grain boundary transitions in binary alloys." Phys. Rev. Lett. 97, (2006).

27. Gao, Q. and Widom, M. "First-principles study of bismuth films at transition-metal grain boundaries." Phys. Rev. B 90, 144102, (2014).

28. Straumal, B. et al. "Wetting and premelting of triple junctions and grain boundaries in the Al-Zn alloys." Materials Science and Engineering: A 495, 126-131, (2008).

29. Luo, J. "Developing Interfacial Phase Diagrams for Applications in Activated Sintering and Beyond: Current Status and Future Directions." J. Am. Ceram. Soc. 95, 2358-2371, (2012).

30. Luo, J., Gupta, V. K., Yoon, D. H. and Meyer, H. M. "Segregation-induced grain boundary premelting in nickel-doped tungsten." Appl. Phys. Lett. 87, (2005).

31. Shi, X. M. and Luo, J. "Grain boundary wetting and prewetting in Ni-doped Mo." Appl. Phys. Lett. 94, 251908, (2009).

32. Weissmuller, J. "Alloy Effects in Nanostructures." Nanostruct. Mater. 3, 261-272, (1993).

33. Koch, C. C., Scattergood, R. O., Darling, K. A. and Semones, J. E. "Stabilization of nanocrystalline grain sizes by solute additions." J. Mater. Sci. 43, 7264-7272, (2008).

34. Koch, C. C., Scattergood, R. O., Saber, M. and Kotan, H. "High temperature stabilization of nanocrystalline grain size: Thermodynamic versus kinetic strategies." J. Mater. Res. 28, 1785-1791, (2013).

35. $\quad$ Weissmuller, J. "Alloy Thermodynamics in Nanostructures." J. Mater. Res. 9, 4-7, (1994).

36. Trelewicz, J. R. and Schuh, C. A. "Grain boundary segregation and thermodynamically stable binary nanocrystalline alloys." Phys. Rev. B 79, (2009).

37. Saber, M., Kotan, H., Koch, C. C. and Scattergood, R. O. "Thermodynamic stabilization of nanocrystalline binary alloys." J. Appl. Phys. 113, 063515, (2013).

38. Murdoch, H. A. and Schuh, C. A. "Stability of binary nanocrystalline alloys against grain growth and phase separation." Acta Mater. 61, 2121-2132, (2013).

39. Zhou, N. and Luo, J. "Developing thermodynamic stability diagrams for equilibriumgrain-size binary alloys." Mater. Lett. 115, 268-271, (2014). 
40. Darling, K. A., Tschopp, M. A., VanLeeuwen, B. K., Atwater, M. A. and Liu, Z. K. "Mitigating grain growth in binary nanocrystalline alloys through solute selection based on thermodynamic stability maps." Comput. Mater. Sci. 84, 255-266, (2014).

41. Wynblatt, P. and Chatain, D. "Anisotropy of segregation at grain boundaries and surfaces." Metallurgical and Materials Transactions A 37, 2595-2620, (2006).

42. Dillon, S. J., Harmer, M. P. and Rohrer, G. S. "The Relative Energies of Normally and Abnormally Growing Grain Boundaries in Alumina Displaying Different Complexions." J. Am. Ceram. Soc. 93, 1796-1802, (2010).

43. Plimpton, S. "Fast Parallel Algorithms for Short-Range Molecular Dynamics." Journal of Computational Physics 117, 1-19, (1995).

44. Mendelev, M. I. et al. "Development of suitable interatomic potentials for simulation of liquid and amorphous Cu-Zr alloys." Philos. Mag. 89, 967-987, (2009).

45. Sadigh, B. et al. "Scalable parallel Monte Carlo algorithm for atomistic simulations of precipitation in alloys." Phys. Rev. B 85, 184203, (2012).

46. Pan, Z. and Rupert, T. J. "Effect of grain boundary character on segregation-induced structural transitions." Phys. Rev. B 93, 134113, (2016).

47. Frolov, T., Olmsted, D. L., Asta, M. and Mishin, Y. "Structural phase transformations in metallic grain boundaries." Nature Communications 4, (2013).

48. Jang, D. and Atzmon, M. "Grain-boundary relaxation and its effect on plasticity in nanocrystalline Fe." J. Appl. Phys. 99, 7, (2006).

49. $\quad$ Ranganathan, S., Divakar, R. and Raghunathan, V. S. "Interface structures in nanocrystalline materials." Scr. Mater. 44, 1169-1174, (2001).

50. Wu, X. L. and Zhu, Y. T. "Partial-dislocation-mediated processes in nanocrystalline Ni with nonequilibrium grain boundaries." Appl. Phys. Lett. 89, 3, (2006).

51. Frolov, T., Divinski, S. V., Asta, M. and Mishin, Y. "Effect of Interface Phase Transformations on Diffusion and Segregation in High-Angle Grain Boundaries." Phys. Rev. Lett. 110, 255502, (2013).

52. Nie, J. F., Zhu, Y. M., Liu, J. Z. and Fang, X. Y. "Periodic Segregation of Solute Atoms in Fully Coherent Twin Boundaries." Science 340, 957-960, (2013).

53. Luo, J. and Shi, X. M. "Grain boundary disordering in binary alloys." Appl. Phys. Lett. 92, (2008).

54. Khalajhedayati, A. and Rupert, T. J. "High-Temperature Stability and Grain Boundary Complexion Formation in a Nanocrystalline Cu-Zr Alloy." JOM, In Press, (2015).

55. Schölhammer, J., Baretzky, B., Gust, W., Mittemeijer, E. and Straumal, B. "Grain Boundary Grooving as an Indicator of Grain Boundary Phase Transformations." Interface Sci. 9, 43-53, (2001).

56. Tai, K., Feng, L. and Dillon, S. J. "Kinetics and thermodynamics associated with Bi adsorption transitions at $\mathrm{Cu}$ and Ni grain boundaries." J. Appl. Phys. 113, 193507, (2013).

57. Gupta, D. "Influence of solute segregation on grain-boundary energy and self-diffusion." Metall. Trans. A 8, 1431-1438, (1977).

58. Browning, N. D., Chisholm, M. F. and Pennycook, S. J. "Atomic-resolution chemical analysis using a scanning transmission electron microscope." Nature 366, 143-146, (1993).

59. D'Alfonso, A. J., Freitag, B., Klenov, D. and Allen, L. J. "Atomic-resolution chemical mapping using energy-dispersive x-ray spectroscopy." Phys. Rev. B 81, 100101, (2010). 
60. Gault, B. et al. "Spatial Resolution in Atom Probe Tomography." Microsc. Microanal. 16, 99-110, (2010).

61. Araullo-Peters, V. J. et al. "A new systematic framework for crystallographic analysis of atom probe data." Ultramicroscopy 154, 7-14, (2015).

62. Araullo-Peters, V. J. et al. "Atom probe crystallography: Atomic-scale 3-D orientation mapping." Scr. Mater. 66, 907-910, (2012).

63. Herbig, M. et al. "Atomic-Scale Quantification of Grain Boundary Segregation in Nanocrystalline Material." Phys. Rev. Lett. 112, 126103, (2014).

64. Schäfer, J., Stukowski, A. and Albe, K. "On the hierarchy of deformation processes in nanocrystalline alloys: Grain boundary mediated plasticity vs. dislocation slip." J. Appl. Phys. 114, 143501, (2013).

65. Panzarino, J. F. and Rupert, T. J. "Tracking Microstructure of Crystalline Materials: A Post-Processing Algorithm for Atomistic Simulations." JOM 66, 417-428, (2014).

66. $\mathrm{Xu}, \mathrm{T}$. and $\mathrm{Li}, \mathrm{M}$. "Geometric methods for microstructure rendition and atomic characterization of poly- and nano-crystalline materials." Philos. Mag. 90, 2191-2222, (2010).

67. Valiev, R. Z., Alexandrov, I. V., Zhu, Y. T. and Lowe, T. C. "Paradox of strength and ductility in metals processed by severe plastic deformation." J. Mater. Res. 17, 5-8, (2002).

68. Lu, K. "Making strong nanomaterials ductile with gradients." Science 345, 1455-1456, (2014).

69. Wei, Y. et al. "Evading the strength-ductility trade-off dilemma in steel through gradient hierarchical nanotwins." Nat Commun 5, (2014).

70. Wu, X., Jiang, P., Chen, L., Yuan, F. and Zhu, Y. T. "Extraordinary strain hardening by gradient structure." Proc. Natl. Acad. Sci. USA 111, 7197-7201, (2014).

71. Lu, L., Chen, X., Huang, X. and Lu, K. "Revealing the Maximum Strength in Nanotwinned Copper." Science 323, 607-610, (2009).

72. Lu, L., Shen, Y. F., Chen, X. H., Qian, L. H. and Lu, K. "Ultrahigh strength and high electrical conductivity in copper." Science 304, 422-426, (2004).

73. Dao, M., Lu, L., Shen, Y. F. and Suresh, S. "Strength, strain-rate sensitivity and ductility of copper with nanoscale twins." Acta Mater. 54, 5421-5432, (2006).

74. Sanders, P. G., Eastman, J. A. and Weertman, J. R. "Elastic and tensile behavior of nanocrystalline copper and palladium." Acta Mater. 45, 4019-4025, (1997).

75. Van Swygenhoven, H., Derlet, P. M. and Froseth, A. G. "Nucleation and propagation of dislocations in nanocrystalline fcc metals." Acta Mater. 54, 1975-1983, (2006).

76. Schiotz, J. and Jacobsen, K. W. "A maximum in the strength of nanocrystalline copper." Science 301, 1357-1359, (2003).

77. Brandl, C., Derlet, P. M. and Van Swygenhoven, H. "Dislocation mediated plasticity in nanocrystalline Al: the strongest size." Modell. Simul. Mater. Sci. Eng. 19, (2011).

78. Asaro, R. J. and Suresh, S. "Mechanistic models for the activation volume and rate sensitivity in metals with nanocrystalline grains and nano-scale twins." Acta Mater. 53, 3369-3382, (2005).

79. Rupert, T. J., Trenkle, J. C. and Schuh, C. A. "Enhanced solid solution effects on the strength of nanocrystalline alloys." Acta Mater. 59, 1619-1631, (2011).

80. Rupert, T. J. "Solid solution strengthening and softening due to collective nanocrystalline deformation physics." Scr. Mater. 81, 44-47, (2014). 
81. Bitzek, E., Brandl, C., Weygand, D., Derlet, P. M. and Van Swygenhoven, H. "Atomistic simulation of a dislocation shear loop interacting with grain boundaries in nanocrystalline aluminium." Modell. Simul. Mater. Sci. Eng. 17, 055008, (2009).

82. Pan, Z. and Rupert, T. J. "Damage nucleation from repeated dislocation absorption at a grain boundary." Comput. Mater. Sci. 93, 206-209, (2014).

83. Farkas, D., Van Petegem, S., Derlet, P. M. and Van Swygenhoven, H. "Dislocation activity and nano-void formation near crack tips in nanocrystalline Ni." Acta Mater. 53, 3115-3123, (2005).

84. Khalajhedayati, A. and Rupert, T. J. "Emergence of localized plasticity and failure through shear banding during microcompression of a nanocrystalline alloy." Acta Mater. 65, 326-337, (2014).

85. Luo, J., Cheng, H., Asl, K. M., Kiely, C. J. and Harmer, M. P. "The Role of a Bilayer Interfacial Phase on Liquid Metal Embrittlement." Science 333, 1730-1733, (2011).

86. Sigle, W., Richter, G., Ruhle, M. and Schmidt, S. "Insight into the atomic-scale mechanism of liquid metal embrittlement." Appl. Phys. Lett. 89, (2006).

87. Madhav Reddy, K. et al. "Enhanced mechanical properties of nanocrystalline boron carbide by nanoporosity and interface phases." Nat Commun 3, 1052, (2012).

88. Pan, Z. and Rupert, T. J. "Amorphous intergranular films as toughening structural features." Acta Mater. 89, 205-214, (2015).

89. Wang, Y. M., Li, J., Hamza, A. V. and Barbee, T. W. "Ductile crystalline-amorphous nanolaminates." Proc. Natl. Acad. Sci. USA 104, 11155-11160, (2007).

90. Brandl, C., Germann, T. C. and Misra, A. "Structure and shear deformation of metallic crystalline-amorphous interfaces." Acta Mater. 61, 3600-3611, (2013).

91. Khalajhedayati, A., Pan, Z. and Rupert, T. J. "Manipulating the interfacial structure of nanomaterials to achieve a unique combination of strength and ductility." Nature Communications 7, 10802, (2016).

92. Heuer, J. K., Okamoto, P. R., Lam, N. Q. and Stubbins, J. F. "Disorder-induced melting in nickel: implication to intergranular sulfur embrittlement." J. Nucl. Mater. 301, 129141, (2002).

93. Chen, H.-P. et al. "Embrittlement of Metal by Solute Segregation-Induced Amorphization." Phys. Rev. Lett. 104, 155502, (2010).

94. Gupta, V. K., Yoon, D. H., Meyer, H. M. and Luo, J. "Thin intergranular films and solidstate activated sintering in nickel-doped tungsten." Acta Mater. 55, 3131-3142, (2007).

95. Messmer, R. P. and Briant, C. L. "The role of chemical bonding in grain boundary embrittlement." Acta Metall. 30, 457-467, (1982).

96. Gibson, M. A. and Schuh, C. A. "Segregation-induced changes in grain boundary cohesion and embrittlement in binary alloys." Acta Mater. 95, 145-155, (2015).

97. Shi, Y. and Falk, M. L. "Does metallic glass have a backbone? The role of percolating short range order in strength and failure." Scr. Mater. 54, 381-386, (2006).

98. Conner, R. D., Johnson, W. L., Paton, N. E. and Nix, W. D. "Shear bands and cracking of metallic glass plates in bending." J. Appl. Phys. 94, 904-911, (2003).

99. Zhang, P. et al. "Microstructural evolution, mechanical properties and deformation mechanisms of nanocrystalline $\mathrm{Cu}$ thin films alloyed with Zr." Acta Mater. 76, 221-237, (2014).

100. Vo, N. Q. et al. "Reaching theoretical strengths in nanocrystalline $\mathrm{Cu}$ by grain boundary doping." Scr. Mater. 65, 660-663, (2011). 
101. Ozerinc, S. et al. "Grain boundary doping strengthens nanocrystalline copper alloys." Scr. Mater. 67, 720-723, (2012).

102. Li, Y. et al. "Segregation Stabilizes Nanocrystalline Bulk Steel with Near Theoretical Strength." Phys. Rev. Lett. 113, 106104, (2014). 
Amorphous Intergranular Film
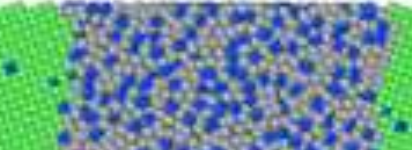

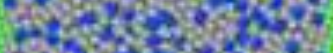

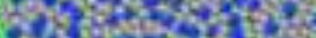

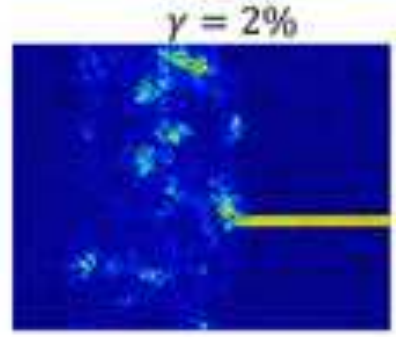
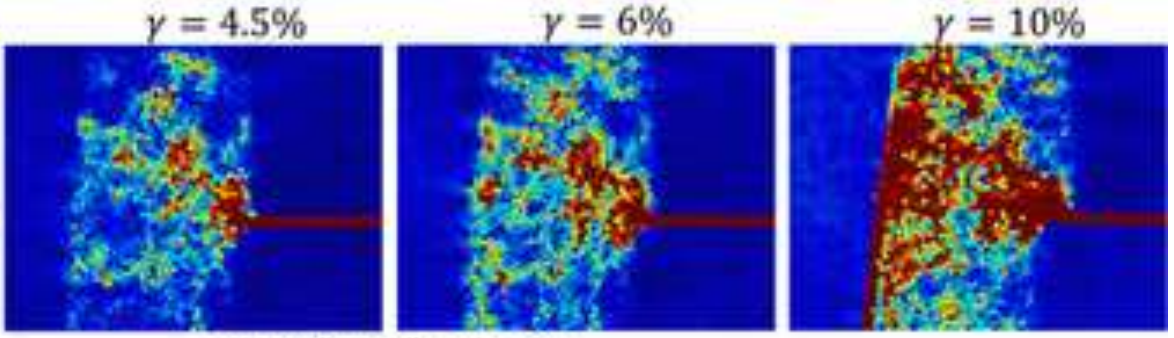

von Mises Shear Strain 